\title{
Marijuana Induced Gingival Enlargement
}

\author{
Dr. Robins Dhakal, ${ }^{1}$ Dr. Shashi Kant Chaudhary, ${ }^{1}$ Dr. Ripu Singh, ${ }^{1}$ Dr. Shivalal Sharma, ${ }^{1}$ \\ Dr. Ashish Shrestha ${ }^{2}$ \\ ${ }^{1}$ Department of Periodontology and Oral Implantology, College of Dental Surgery, \\ BP Koirala Institute of Health Sciences, Dharan, Nepal; \\ ${ }^{2}$ Department of Oral Pathology, College of Dental Surgery, BP Koirala Institute of Health Sciences, Dharan, Nepal.
}

\begin{abstract}
Different aetiologies with various clinical presentations are evident in cases of gingival enlargement. We report a case of 34-year-old male who presented with the chief complaint of gingival overgrowth in upper and lower front teeth region. Patient was systemically healthy and gave no history of common medications known to cause gingival enlargement. He had a history of marijuana consumption for two consecutive years following which gingival swelling increased in severity. Based on clinical and histopathologic findings, provisional diagnosis of combined inflammatory + marijuana induced gingival enlargement was made. Treatment consisted of scaling and root planing followed by sextant wise gingivectomy. Results were stable until three months follow-up. Signs of inflammation were evident when the patient reported after 1.5 years.
\end{abstract}

Keywords: Gingival enlargement; gingivectomy; marijuana.

\section{INTRODUCTION}

The increase in size of the gingiva is termed as gingival enlargement or gingival overgrowth. Gingival enlargement has multiple etiologies and can exhibit different clinical manifestations. ${ }^{1}$ Common forms can be attributed to usage of medications like phenytoin, amlodipine, and cyclosporine. Other causes could be conditioned, idiopathic and neoplastic enlargements. The cases of marijuana induced gingival enlargement are rarely reported - and if at all - reported insufficiently in the literature. Detailed history followed by clinical, laboratory and histopathologic examination is mandatory for a definitive diagnosis. Cannabidiol, a component of marijuana, which shares similar structure with phenytoin, is thought to produce gingival enlargement with manifestations similar to that of phenytoin. ${ }^{2}$ For any type of gingival enlargement, the procedural plan of initial treatment encompasses scaling and root planing, followed by gingivectomy, if required. However, recurrences are common, so the patients are kept at regular follow-ups.

\footnotetext{
Correspondence:

Dr. Robins Dhakal

Department of Periodontology and Oral Implantology, College of Dental Surgery, BP Koirala Institute of Health Sciences, Dharan, Nepal.

email: robinsdarock123@gmail.com

Citation

Dhakal R, Chaudhary SK, Singh $R$, Sharma S, Shrestha A. Marijuana Induced Gingival Enlargement. J Nepal Soc Perio Oral Implantol. 2019;3(5):25-8
}

\section{CASE REPORT}

A 34-year-old male presented to the Department of Periodontology and Oral Implantology, BP Koirala Institute of Health Sciences, with the chief complaint of gingival overgrowth in upper and lower teeth region for three years. The overgrowth progressively increased in size which was associated with bleeding while brushing and patient had difficulty in mastication. He gave no history of any systemic medications that were known to cause gingival enlargement and was systemically healthy. However, patient had the habit of consuming marijuana almost daily for two years in the wake of which the enlargement exacerbated assisted by the frequency of consumption. As reported, it had been five to six months since the patient had abstained from marijuana smoking. Alongside marijuana, the patient also reported to have been a tobacco smoker with a sustained consumption frequency of four to five sticks continuously for four to five years. Mouth breathing was not reported.

Intraoral examination revealed generalised gingival enlargement which was diffuse, fibrotic, pebbled and nodular (Figure 1a). The enlargement was comparatively severe in the maxillary and mandibular anterior sextants than posterior (Figure 1b). The buccal or labial portion was more severe than the palatal or lingual portion. The enlargement covered three quarters or more of the crown in both upper and lower arches. Inflammatory component was also present which was evident by the presence of local factors. Generalised pseudo pockets were present. No pronounced attachment loss and 


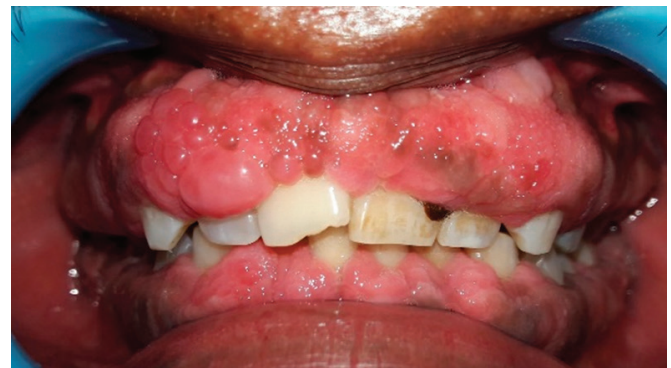

Figure 1(a): Pre-operative Frontal view.

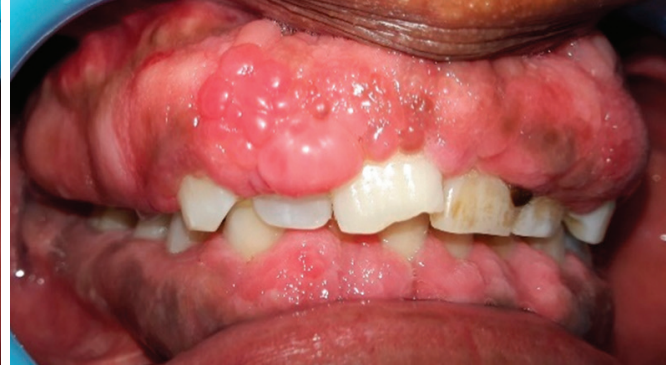

Figure 1(b): Right lateral view.

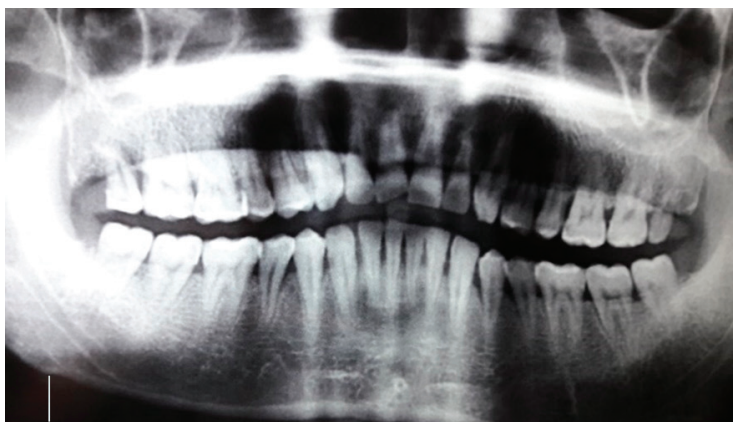

Figure 2: Orthopantomogram.

mobility was seen. Slight mesiolabial rotation of 11 was seen. On radiographic examination, significant bone loss was not evident (Figure 2).

A provisional diagnosis of combined gingival enlargement (inflammatory + marijuana induced) was made as the evidence was inconclusive to reach a confirmatory diagnosis. Idiopathic gingival enlargement, hereditary gingival fibromatosis, and leukemic gingival enlargement were kept under differential diagnosis. The laboratory tests were within normal limits and serological tests were negative. The patient was briefed with the information regarding the type of disease, its course, severity, treatment plan and significance of further maintenance visits. Scaling and root planing were performed in the initial visit as a part of phase I therapy. After two weeks, subgingival scaling was done and, after 1-month sextant wise gingivectomy was performed.
Surgical procedure: After adequate anaesthesia with 2\% lignocaine and 1:200000 adrenaline, external bevel gingivectomy as described by Goldman was performed 1st in the upper anterior sextant (Figure 3a). External bevel gingivectomy was preferred as there was presence of pseudo pockets and absence of intrabony defects. ${ }^{1}$ Kirkland gingivectomy knife, Bard-Parker blade no 15 and tissue nippers were used (Figure 3b). After two weeks, lower anterior sextant (Figure 3c) was treated followed by upper right posterior sextant (Figure 3d). The tissue excised was sent for histopathologic examination. Periodontal dressing was placed (Figure 3e).

Histopathological report (Figure 4): It revealed hyperplastic parakeratotic stratified squamous epithelium with irregular rete ridges. Connective tissue was fibrous along with dense collagen bundles. Numerous endothelial cells lined blood

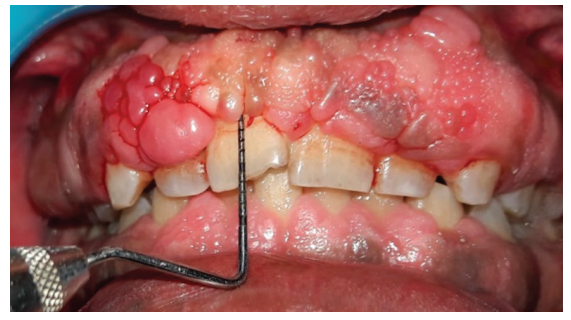

Figure 3(a): Marking of pockets for external bevel gingivectomy.

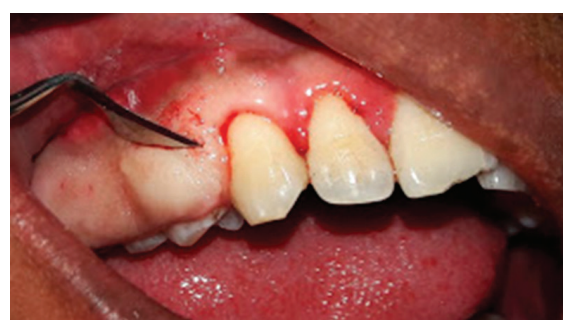

Figure 3(d): Right upper posterior

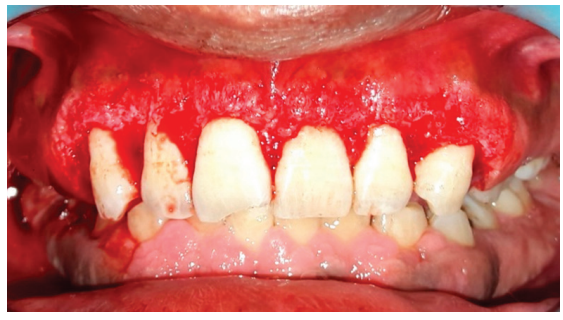

Figure 3(b): Upper anterior after gingivectomy and gingivoplasty.

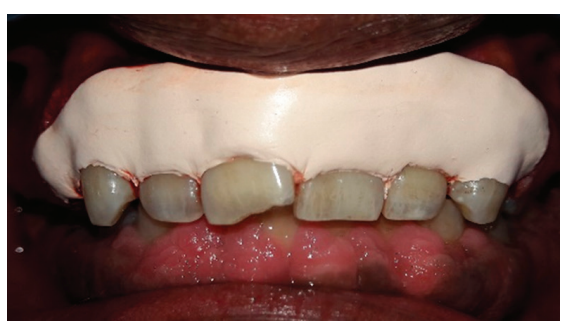

Figure 3(e): Coe pak.

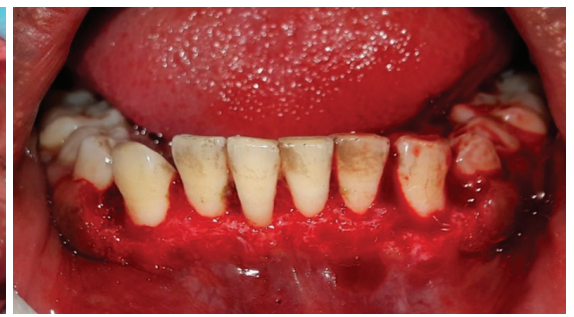

Figure 3(c): Lower anterior.

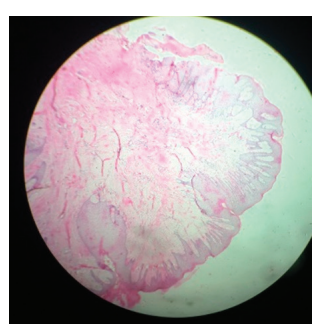

Figure 4: Histopathological slide (Hematoxylin and Eosin; 10 X). 


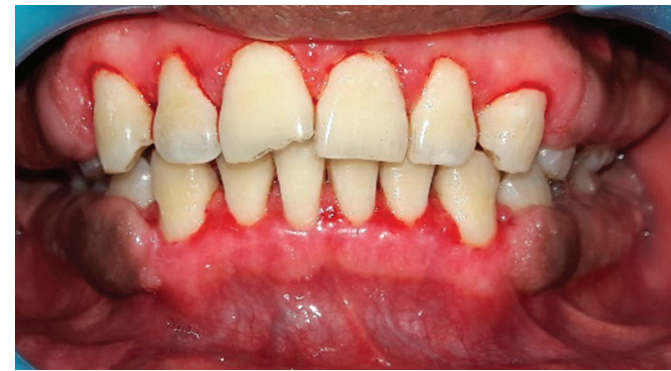

Figure 5(a): After 1 month.

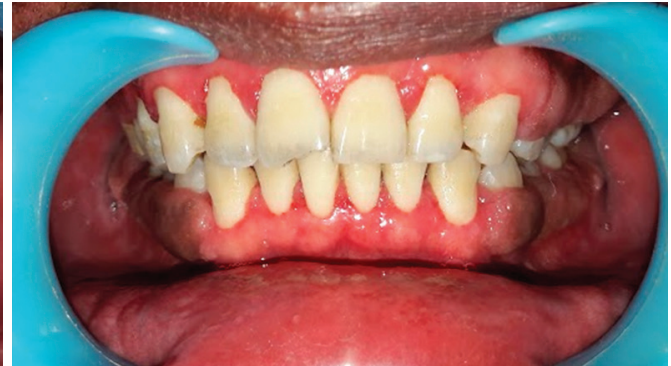

Figure 5(b): after 3 months.

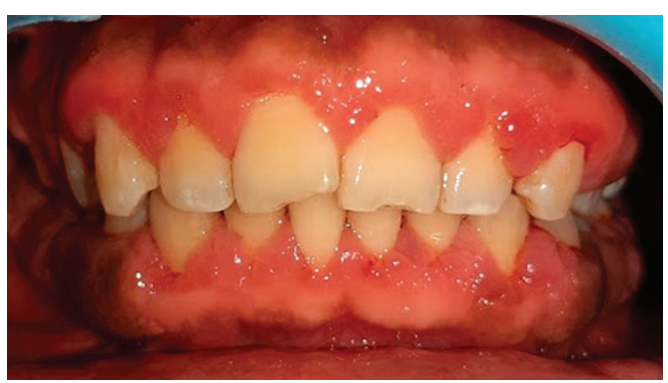

Figure 6: After 1.5 years after scaling.

vessels along with sparse inflammatory cells infiltrate was seen. Predominantly lymphocytes were evident.

Post-operative care: Ibuprofen $400 \mathrm{mg}$ twice daily for two days was given. Chlorhexidine mouthwash $0.2 \%$ twice daily for two weeks was prescribed. Oral hygiene instructions were provided. Periodontal dressing was removed after two weeks. The patient was advised to abstain from all sorts of tobacco and marijuana oral consumption. The postoperative evaluation was done at one (Figure 5a) and 3 (Figure 5b) months after surgery. The patient re-visited only after 1.5 years. Signs of inflammation were seen since the patient had not maintained his oral hygiene and had continued to smoke but with less frequency as before (Figure 6 after scaling).

\section{DISCUSSION}

Cannabis preparations are largely derived from the female plant of Cannabis sativa. The primary psychoactive constituent is tetrahydrocannabinol (THC). The THC content is highest in the flowering tops, declining in the leaves, lower leaves, stems, and seeds of the plant. Marijuana (THC content $0 \cdot 5-5 \cdot 0 \%$ is prepared from the dried flowering tops and leaves. ${ }^{3}$ The association between chronic marijuana use and gingival enlargement was first noted by Layman and colleagues ${ }^{4}$ which showed generalised, fieryred enlargement resembling that of phenytoin. A study by Rawal and colleagues ${ }^{2}$ suggested that the Cannabidiol may promote fibrotic gingival enlargement by increasing gingival fibroblast production of transforming growth factor beta and fibronectin while decreasing matrix metalloproteinase production and activity. It exhibits pharmacological effects, including antiepileptic properties that are comparable to those of phenytoin, and both drugs fulfil the stereochemical requirements for antiepileptic drug action. So the underlying pathogenetic mechanism might be similar to phenytoininduced. ${ }^{2}$

In our case, the presentation was nodular or pebbled type, more severe in the maxillary and mandibular anterior sextants which were similar to that of Rawal and Layman. ${ }^{4}$ Baddour and colleagues ${ }^{4}$ reported alveolar bone loss and severe mobility of mandibular molars which was not present in our case. The histopathological report of our case showed hyperplastic epithelium with numerous collagen bundles but sparse inflammatory infiltrate. It resembled that found in cases of phenytoin-induced and gingival fibromatosis cases. ${ }^{1}$ Leukemic type of gingival enlargement was ruled out as the hematological investigations were normal and there were no such clinical features. There was no familial history of such enlargement when enquired. According to Gorlin et al, hereditary gingival fibromatosis is most commonly associated with hypertrichosis, mental retardation, and epilepsy which were not present in our case. ${ }^{5}$ Idiopathic gingival enlargement is a diagnosis of exclusion when there is no evidence of any cause ${ }^{6}$ and there are no contributory medical or family history. ${ }^{7}$ With no cause, it might be idiopathic but based on history and no recurrences, the case might be a marijuana induced gingival enlargement. Further studies and research are warranted for conclusive evidence.

\section{SUMMARY}

The literature is scarce reporting marijuana induced gingival enlargement; therefore, enough shreds of evidence are required to reach a conclusive remark to differentiate it from possible idiopathic gingival enlargement. However, this case report might be useful in raising awareness among fellow colleagues and cannabis users regarding its effect on periodontal health. 


\section{ACKNOWLEDGEMENT}

Dr. Khushboo Goel, Dr. Sajeev Shrestha, and Dr. Pujan Acharya, Department of Periodontology and Oral Implantology, College of Dental Surgery, BPKIHS, Dharan.

\section{REFERENCES}

1. Carranza, F.A., Newman, M.G., Takei, H.H. \& Klokkevold PR. Carranza's Clinical Periodontology. 12th ed. Saunders, Elsevier; 2015.

2. Rawal SY, Dabbous MKh, Tipton DA. Effect of cannabidiol on human gingival fibroblast extracellular matrix metabolism: MMP production and activity, and production of fibronectin and transforming growth factor Q. J Periodont Res. 2012;47:320-9.

3. Hall W, Solowij N. Adverse effects of cannabis. Lancet. 1998;352:1611-6.

4. Rawal SY, Tatakis DN, Tipton DA. Periodontal and oral manifestations of marijuana use. J Tennessee Dent Assoc.2012;92:26-31.

5. Baptista IP: Hereditary gingival fibromatosis: a case report. J Clin Periodontol. 2002;29:871-4.

6. Ko YCK, Farr JB, Yoon A and Philipone E. Idiopathic Gingival Fibromatosis: Case Report and Review of the Literature. Am J Dermatopathol. 2016;38:e68-e71.

7. Lai B, Muenzer J, Roberts MW. Idiopathic Gingival Hyperplasia: A case eport with a 17-year follow up. Case Rep Dent. $2011 ; 2011: 986237$. 